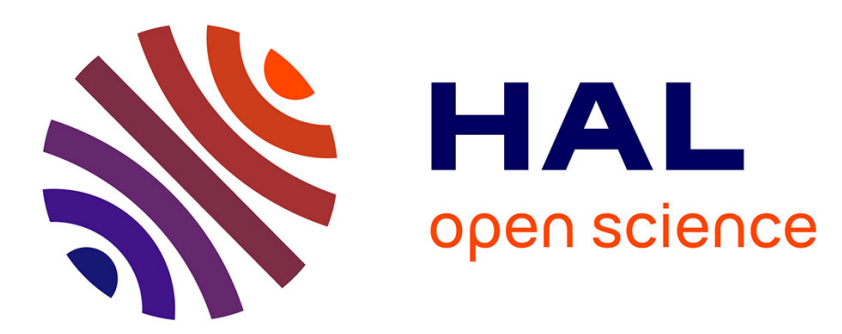

\title{
A Stochastic Geometry Based Approach to Tractable 5G RNPO with a New H-LOS Model
}

\author{
Yassine Hmamouche, Mustapha Benjillali, Samir Saoudi
}

\section{To cite this version:}

Yassine Hmamouche, Mustapha Benjillali, Samir Saoudi. A Stochastic Geometry Based Approach to Tractable 5G RNPO with a New H-LOS Model. WCNC 2019: IEEE Wireless Communications and Networking Conference, Apr 2019, Marrakech, Morocco. 10.1109/WCNC.2019.8885422 . hal02067720

\section{HAL Id: hal-02067720 \\ https://imt-atlantique.hal.science/hal-02067720}

Submitted on 14 Mar 2019

HAL is a multi-disciplinary open access archive for the deposit and dissemination of scientific research documents, whether they are published or not. The documents may come from teaching and research institutions in France or abroad, or from public or private research centers.
L'archive ouverte pluridisciplinaire HAL, est destinée au dépôt et à la diffusion de documents scientifiques de niveau recherche, publiés ou non, émanant des établissements d'enseignement et de recherche français ou étrangers, des laboratoires publics ou privés. 


\title{
A Stochastic Geometry Based Approach to Tractable 5G RNPO with a New $H$-LOS Model
}

\author{
Yassine Hmamouche ${ }^{1,2}$, Mustapha Benjillali ${ }^{2}$, and Samir Saoudi ${ }^{1}$ \\ ${ }^{1}$ IMT Atlantique, Lab-STICC, UBL, 29238 Brest, France \\ Emails: \{yassine.hmamouche; samir.saoudi\}@imt-atlantique.fr \\ ${ }^{2}$ Communication Systems Department, INPT, Rabat, Morocco \\ Email: benjillali@ieee.org
}

\begin{abstract}
We consider a 3D cellular network in which generalized shadowing and radio network planning and optimisation (RNPO) parameters (e.g., antenna height, antenna tilt/azimuth, power biaising...) are incorporated into the cell-selection model. Using tools from stochastic geometry (SG), we derive an equivalent 2D network in which no shadowing and RNPO parameters are considered. Next, we derive coverage probability for a tractable case-study network, and the regimes where coverage probability is maximized in addition to the interferencelimited one are investigated. An intermediary result is a closedform expression generator encompassing the $\mathcal{Q}$-function basedexpression in [1]. Numerical results confirm the accuracy of our approximations.
\end{abstract}

\section{INTRODUCTION}

With the ongoing proliferation of data-hungry devices and applications, data traffic volumes in the coming years are expected to be multi-fold higher compared to today's levels. One way to tackle this challenge is by deploying ultra-dense networks (UDNs) [2]. However, densification will result in large coverage overlap areas, which increases the risk of othercell interference and then reduces the network performance and system capacity. Consequently, environment characteristics such as shadowing, and RNPO parameters such as antenna height [3], antenna tilt/azimuth angle [4]- [6] and transmit power biaising [7] are strongly required for the analysis of UDNs performance since they affect directly the probability of line-of-sight (LOS) and non-line-of-sight (NLOS) connections and then cells overlaping.

Due to its tractability and ability to capture spatial averages, SG has emerged as a potential mathematical tool for modeling cellular networks [1], [3]- [7]. In fact, by considering a standard path-loss model and ignoring shadowing and any RNPO parameter effect, the seminal work in [1] provides comprehensive understanding about the behavior of UDNs performance. An important outcome is the signal-to-interference-plus-noise ratio (SINR) invariance property, which states that the SINR increases almost linearly with base station (BS) density to the point where noise becomes negligible; after which SINR remains stable and independent from BS density. However, using standard path-loss model and ignoring RNPO parameters in more realistic scenarios has raised some limitations [8],

This work is funded by a research grant from PRACOM and the Regional Council of Brittany, France. calling for an imperative revisitation of the model. Authors of [9] proved that the SINR invariance property is no longer valid when using the dual-slope path-loss model. A similar effect is reported in [3] for elevated BSs, and in [4] for a network using non-directional antennas.

The motivation behind this paper is then to find a tractable manner to study UDNs performance when incorporating generalized shadowing and RNPO parameters into the cellselection model. Using tools from SG, we first $i$ ) develop a 3D-2D network equivalence where a 3D network with shadowing and RNPO parameters is stochastically equivalent to a 2D network in which they are not considered. Next, for mathematical convenience, ii) we focus our analysis on a tractable case-study in which shadowing and RNPO parameters are captured via aggregated parameters related with LOS and NLOS connections. iii) The coverage probability is then computed confirming that our expression is general enough to accommodate several previous expressions. Next, iv) we investigate the scaling law of the BS density that maximizes network performance as well as the coverage probability in the interference-limited regime.

The following notation will be used throughout the paper. $\mathbb{P}\{$.$\} and \mathbb{E}\{$.$\} stand for the probability and expectation$ measure. $\mathbb{L}_{X}(s)=\mathbb{E}\left\{\mathrm{e}^{-s X}\right\}$ is the Laplace transform of a random variable $X$ evaluated at $s$. We define for any reals $m, x \in \mathbb{R}, F_{m}(x)={ }_{2} F_{1}(1, m ; m+1 ;-x)$ where ${ }_{2} F_{1}(., . ; ; ; z)$ is the Gauss hypergeometric function. $g^{-1}($.$) is the inverse$ function of a function $g($.$) .$

\section{SYSTEM MODEL AND THE PATH LOSS PROCESS WITH SHADOWING AND RNPO PARAMETERS (PLPSR)}

\section{A. System Model}

We consider a downlink cellular network, in which BSs are scattered randomly according to a homogeneous PPP $\Phi_{b} \in \mathbb{R}^{3}$ with density $\lambda_{b}$. We assume that each BS is equipped with directional antennas, has at least one connected user and transmits with a fixed power $P_{\mathrm{tx}}$. Denote $\sigma^{2}$ the variance of the additive noise and SNR $=P_{\mathrm{tx}} / \sigma^{2}$. We consider a realization of RNPO parameters of interest: BS antenna elevation height parametrized by a random variable $\xi_{x_{h}}$, electrical/mechanical antenna tilt angle by $\xi_{x_{t}}$, antenna azimut angle by $\xi_{x_{a}}$ and range expansion (RE) biais by $\xi_{x_{b}}$. For each BS $x \in \Phi_{b}$, 
we add independent ${ }^{1}$ marks $\left(h_{x}, \chi_{x}, \xi_{x}, \alpha_{x}, T_{x}\right)$, where for the link between $x$ and the typical user located at $O, h_{x}$ denote the small scale fading assumed to be exponentially distributed with unit mean, $\chi_{x}$ is the shadowing effect assumed to be arbitrarily distributed, $\alpha_{x}$ is the path-loss exponent, $T_{x}$ is the SINR threshold of $x$, and $\xi_{x}$ is the vector $\xi_{x}=\left(\xi_{x_{h}}, \xi_{x_{t}}, \xi_{x_{a}}, \xi_{x_{b}}\right)$ of RNPO parameters, such as the received power at $O$ from the BS $x \in \Phi_{b}$ is

$$
P_{\mathrm{rx}}=\frac{\chi_{x} h_{x} P_{\mathrm{tx}}}{\left(\Psi\left(r_{x} ; \alpha_{x} ; \xi_{x}\right)\right)^{\alpha_{x}}},
$$

where $r_{x}$ is the horizontal distance between $x$ and $O$, and $\Psi($.$) is a generalized function to capture RNPO parameters$ combined with the path-loss function. If there is such a function, it is reasonable to require of it the following properties: (i) monotonically increasing such as $\Psi\left(0 ; . ; \xi_{x_{h}}=0^{2}\right)=\Psi_{0} \geq 1$ at the origin $O$, this is in order to cover realistic bounded path-loss models and ensure that the received power cannot exceed the transmitted one, (ii) $\Psi\left(r_{x} ; . ; \xi_{x}\right) \equiv \Psi\left(r ; . ; \xi_{x}^{\prime}\right)$ such as $r=\sqrt{r_{x}^{2}+\xi_{x_{h}}^{2}}$ and $\xi_{x}^{\prime}$ is the vector $\xi_{x}^{\prime}=\left(\xi_{x_{t}}, \xi_{x_{a}}, \xi_{x_{b}}\right)$, (iii) the mean value of the shot noise process is finite, i.e., from the Campbell's theorem [10, Corollary 1.4.6.], we have

$$
\mathbb{E}\left\{\sum_{x \in \Phi_{b}} P_{\mathrm{rx}}\right\}=\lambda_{b} P_{\mathrm{tx}} \int_{\mathbb{R}^{3}} \frac{\mathbb{E}\left\{\chi_{x}\right\} \mathrm{d} x}{\left(\Psi\left(r_{x} ; \alpha_{x} ; \xi_{x}\right)\right)^{\alpha_{x}}}<\infty,
$$

The marked PPP, will be denoted, with a slight abuse of notation, also as $\Phi_{b}$.

Remark 1. The proposed model is general enough to accommodate various choices of RNPO parameters and pathloss models, e.g., if the power law path-loss is adopted and BS height is the only RNPO parameter considered [3], $\xi_{x}=\xi_{x_{h}}$ captures BSs height and $\Psi\left(r_{x} ; . ; \xi_{x}\right)=$ $\sqrt{r_{x}^{2}+\xi_{x}^{2}}$. When considering also tilt angle [5], azimuth angle [6] and RE biais [7], we have $\Psi\left(r_{x} ; \alpha_{x} ; \xi_{x}\right)=$ $\sqrt{r_{x}^{2}+\xi_{x_{h}}^{2}}\left[G_{\text {tilt }}\left(\xi_{x_{t}}\right) G_{a z i m u t}\left(\xi_{x_{a}}\right) B\left(\xi_{x_{b}}\right)\right]^{\frac{-1}{\alpha_{x}}}$, where $G_{\text {tilt }}($. is the antenna vertical radiation pattern parametrized by $\xi_{x_{t}}, G_{\text {azimut }}($.$) is the antenna horizontal radiation pattern$ parametrized by $\xi_{x_{a}}$ and $B($.$) is the association bias$ parametrized by $\xi_{x_{b}}$.

B. Path Loss process with shadowing and RNPO parameters

We define the path-loss process with shadowing and RNPO parameters (PLPSR) of $\Phi_{b}$, the point process mapped from $\Phi_{b}$ on $\mathbb{R}^{+}$, as

$$
\Sigma=\left\{y=\chi_{x}^{-1 / \alpha_{x}} \Psi\left(r_{x} ; \alpha_{x} ; \xi_{x}\right), x \in \Phi_{b}\right\} .
$$

Moreover, in order to capture the SINR threshold distribution, we consider the following independently marked PLPSR

$$
\Delta=\left\{\left(\Sigma, T_{x}\right), x \in \Phi_{b}\right\} .
$$

The following lemma gives the intensity measure of $\Delta$, which generalizes several previous results in [11] [12].

\footnotetext{
${ }^{1}$ We omit the dependence scenario here, e.g., $\xi_{x}$ and $\alpha_{x}$ may be correlated when a tunning of the RNPO parameters $\xi_{x}$ can impact $\alpha_{x}$ by determining the link nature (LOS or NLOS) between a BS and the typical user.

${ }^{2} \xi_{x} \equiv 0$ is equivalent to no RNPO parameter considered on $x$, i.e., BS antenna is omnidirectional with 0 meter elevation and $B\left(\xi_{b}\right) \equiv 1$.
}

Lemma 1. The point process $\Delta$ is a $1 D$ independently marked PPP on $\mathbb{R}^{+}$with intensity measure

$$
\Lambda_{\Delta}(s, t)=\frac{4 \pi \lambda_{b}}{3} \mathbb{E}\left\{\left[\Psi^{-1}\left(s \chi_{x}^{\frac{1}{\alpha_{x}}} ; \alpha_{x} ; \xi_{x}^{\prime}\right)\right]^{3} \mathbb{1}\left(T_{x} \leq t\right)\right\},
$$

Proof. By the displacement theorem [10, Theorem 1.3.9] and the Campbell's theorem. $\Delta$ is a PPP with intensity measure

$$
\begin{aligned}
& \Lambda_{\Delta}(s, t)=\lambda_{b} \mathbb{E}\left\{\int_{\mathbb{R}^{3}} \mathbb{1}\left(\frac{\Psi\left(r_{x} ; \alpha_{x} ; \xi_{x}\right)}{\chi_{x}^{\frac{1}{\alpha_{x}}}} \leq s, T_{x} \leq t\right) \mathrm{d} x\right\} \\
& \stackrel{(a)}{=} 4 \pi \lambda_{b} \mathbb{E}\left\{\int_{\mathbb{R}^{+}} \mathbb{1}\left(\frac{\Psi\left(r ; \alpha_{x} ; \xi_{x}^{\prime}\right)}{\chi_{x}^{\frac{1}{\alpha_{x}}}} \leq s\right) \mathbb{1}\left(T_{x} \leq t\right) r^{2} \mathrm{~d} r\right\} \\
& =\frac{4 \pi \lambda_{b}}{3} \mathbb{E}\left\{\int_{\mathbb{R}^{+}}\left[\Psi^{-1}\left(s u^{\frac{1}{\alpha_{x}}} ; \alpha_{x} ; \xi_{x}^{\prime}\right)\right]^{3} \mathbb{1}\left(T_{x} \leq t\right) \mathbb{P}_{\chi_{x}}\{\mathrm{~d} u\}\right\} \\
& =\frac{4 \pi \lambda_{b}}{3} \mathbb{E}\left\{\left[\Psi^{-1}\left(s \chi_{x}^{\frac{1}{\alpha_{x}}} ; \alpha_{x} ; \xi_{x}^{\prime}\right)\right]^{3} \mathbb{1}\left(T_{x} \leq t\right)\right\},
\end{aligned}
$$

where (a) follows from the marks independence of the process $\Delta$ and property (ii) of $\Psi($.$) .$

Remark 2. If we assume that $T_{x} \equiv T$ is constant over all BSs of $\Phi_{b}$. It is easy to mention from lemma 1 that for the defined $R N P O$ parameters (Remark 1), $\Delta$ is generally a homogeneous $P P P$ with density

$$
\lambda_{\Delta}(s)=\lim _{t \rightarrow \infty} \frac{1}{4 \pi s^{2}} \frac{\partial \Lambda_{\Delta}(s, t)}{\partial s},
$$

independent from $s$ and proportionally related to $\mathbb{E}\left\{\chi_{x}^{3 / \alpha_{x}}\right\}$, e.g., when considering only height ( $\left.\xi_{x} \equiv \xi_{x_{h}}\right)$, we have $\lambda_{\Delta}=\lambda_{b} \mathbb{E}\left\{\chi_{x}^{3 / \alpha_{x}}\right\}<\infty$.

Definition 1. Similarly to [11, definition 1] and [12, definition 2], a $3 D$ marked PPP $\Phi_{b}$ is said to be equivalent in distribution to a $2 D$ marked $P P P \Phi_{b}^{\prime}$ if they generate the same $1 D$ marked PPP $\triangle$ with the intensity measure $\Lambda(s, t)$.

Proposition 1. The marked process $\Phi_{b} \in \mathbb{R}^{3}$ is stochastically equivalent to a marked $P P P \Phi_{b}^{\prime} \in \mathbb{R}^{2}$ in which shadowing and $R N P O$ parameters are not considered, i.e., $\chi_{x}^{\prime} \equiv 1$ and $\xi_{x}^{\prime} \equiv 0$, and endowed with marks $T_{x}^{\prime} \equiv T_{x}$ whose distribution is

$$
G_{s}^{\prime}(t)=\frac{1}{4 \pi s^{2} \lambda_{\Delta}(s)} \frac{\partial \Lambda_{\Delta}(s, t)}{\partial s},
$$

and the density of $\Phi_{b}^{\prime}$ is expressed as $\lambda_{b}^{\prime}(s)=2 s \lambda_{\Delta}(s)$

Proof. The proof of proposition 1 is analogous to that of [11, proposition 4]. In fact, the intensity measure of $\Delta^{\prime}-$ the independently marked PLPSR of $\Phi_{b}^{\prime}$ - when $\chi_{x}^{\prime} \equiv 1$ and $\xi_{x}^{\prime} \equiv 0$ is

$$
\begin{aligned}
& \Lambda_{\Delta^{\prime}}(v, t)=2 \pi \mathbb{E}\left\{\int_{\mathbb{R}^{+}} \mathbb{1}(u \leq v) \mathbb{1}\left(T_{x}^{\prime} \leq t\right) \lambda_{b}^{\prime}(u) u \mathrm{~d} u\right\} \\
& =2 \pi \int_{0}^{v} G_{u}^{\prime}(t) \lambda_{b}^{\prime}(u) u \mathrm{~d} u \stackrel{(a)}{=} \Lambda_{\Delta}(v, t),
\end{aligned}
$$

where (a) holds if equations (7) and (8) are met.

From Proposition 1, if noise, small scale fading and pathloss exponent are the same, we have then 


$$
\begin{aligned}
\operatorname{SINR}\left(x_{0}\right) & =\left.\frac{\frac{h_{x_{0}} \chi_{x_{0}}}{\left(\Psi\left(r_{x_{0}} ; \alpha_{x_{0}} ; \xi_{x_{0}}\right)\right)^{\alpha_{x}}}}{\sum_{x \in \Phi_{b} \backslash\left\{x_{0}\right\}} \frac{h_{x} \chi_{x}}{\left(\Psi\left(r_{x} ; \alpha_{x} ; \xi_{x}\right)\right)^{\alpha_{x}}}+\left(\frac{1}{\operatorname{SNR}}\right)}\right|_{\lambda_{b}} \\
& \left.\stackrel{(\mathrm{d})}{=} \frac{h_{y_{0}} y_{0}^{-\alpha_{y_{0}}}}{\sum_{y \in \Phi_{b}^{\prime} \backslash\left\{y_{0}\right\}} h_{y} y^{-\alpha_{y}}+\frac{1}{\operatorname{SNR}}}\right|_{\lambda_{b}^{\prime}}=\operatorname{SINR}\left(y_{0}\right),
\end{aligned}
$$

where $\stackrel{(\mathrm{d})}{=}$ denotes equivalence in distribution, $x_{0}=\arg \max _{x \in \Phi_{b}}\left\{P_{\mathrm{tx}} \chi_{x}\left(\Psi\left(r_{x} ; \alpha_{x} ; \xi_{x}\right)\right)^{-\alpha_{x}}\right\} \quad$ and $y_{0}=\arg \max _{y \in \Phi_{b}^{\prime}}\left\{y^{-\alpha_{y}}\right\}$.

\section{A TRACTABLE CASE STUDY}

Now, for mathematical convenience and model tractability, we take a minor detour from studying the stochastic equivalence between a 3D network with shadowing and RNPO parameters and a 2D network where they are absorbed into the model. In fact, we assume that the equivalent PPP $\Phi_{b}^{\prime} \in \mathbb{R}^{2}$ is homogeneous $\lambda_{b}^{\prime}=\lambda$, the SINR target is constant over all BSs $T_{x}^{\prime}=\mathrm{T}$, and the path-loss exponent $\alpha_{x}^{\prime}$ is distancedependent according to the transmission path (LOS or NLOS) between BSs and the typical user, i.e., $\alpha_{x}^{\prime} \in\left\{\alpha_{\text {los }}, \alpha_{\text {nlos }}\right\}$ such as $\eta=\alpha_{\text {nlos }} / \alpha_{\text {los }} \geq 1$. We consider that each BS $x \in \Phi_{b}^{\prime}$ has a LOS path towards the typical user with a LOS probability denoted $P_{\text {los }}$.

\section{A. The H-LOS probability model}

Since common LOS probability functions are build upon exponentially decreasing functions [13] rendering analysis less tractable, we propose to approximate them by the following piece-wise linear model, consistent with the models adopted by 3 GPP [14] and dubbed here the $H$-LOS model,

$$
P_{\text {los }}\left(r_{x}\right)=\left\{\begin{array}{llr}
1 & \text { if } & 0 \leq r_{x} \leq \mathrm{R}_{\text {los }} \\
1-\frac{r_{x}-R_{\text {los }}}{R_{\text {nlos }}-R_{\text {los }}} & \text { if } & \mathrm{R}_{\text {los }} \leq r_{x} \leq \mathrm{R}_{\text {nlos }} \\
0 & \text { if } & r_{x}>\mathrm{R}_{\text {nlos }}
\end{array},\right.
$$

where $\mathrm{R}_{\text {los }}$ is the maximum link distance between a LOS $\mathrm{BS}$ and the typical user such as there are no nearer NLOS BS to the typical user, while $R_{\text {nlos }}$ is the minimum link distance between a NLOS BS and the typical user such as there are no farther LOS BS. Mathematically,

$$
\begin{aligned}
& \mathrm{R}_{\text {los }}=\max _{x \in \Phi_{\text {los }}}\left\{r_{x} ; r_{x}<r_{y}, \forall y \in \Phi_{\text {nlos }}\right\}, \\
& \mathrm{R}_{\text {nlos }}=\min _{y \in \Phi_{\text {nlos }}}\left\{r_{y} ; r_{x}<r_{y}, \forall x \in \Phi_{\text {los }}\right\},
\end{aligned}
$$

such as $\Phi_{\text {los }}$ and $\Phi_{\text {nlos }}$ are the PPPs of LOS and NLOS BSs of $\Phi_{b}^{\prime}$ respectively.

Fig. 1 shows the three regions of the network generated by the $H$-LOS probability model in (11). Note that $\mathrm{R}_{\text {los }}$ and $\mathrm{R}_{\text {nlos }}$ can be expanded by low shadowing effect and/or RNPO actions that expand cells size (uptilt, increasing association biais, azimuth that avoid blockages...). We propose therefore the interpretation that shadowing and RNPO parameters are absorbed into the 2D PPP $\Phi_{b}^{\prime}$, but their impact is still captured via the fluctuation of aggregated parameters $\mathrm{R}_{\mathrm{los}}$ and $\mathrm{R}_{\mathrm{nlos}}$.

The NLOS probability is obtained as $P_{\text {nlos }}\left(r_{x}\right)=1-$ $P_{\text {los }}\left(r_{x}\right), \forall x \in \Phi_{b}^{\prime}$, and the path-loss function as

$$
\mathcal{L}\left(r_{x}\right)= \begin{cases}r_{x}^{-\alpha_{\text {los }}} & \text { with probability } P_{\text {los }}\left(r_{x}\right) \\ K r_{x}^{-\alpha_{\text {nlos }}} & \text { with probability } P_{\text {nlos }}\left(r_{x}\right),\end{cases}
$$

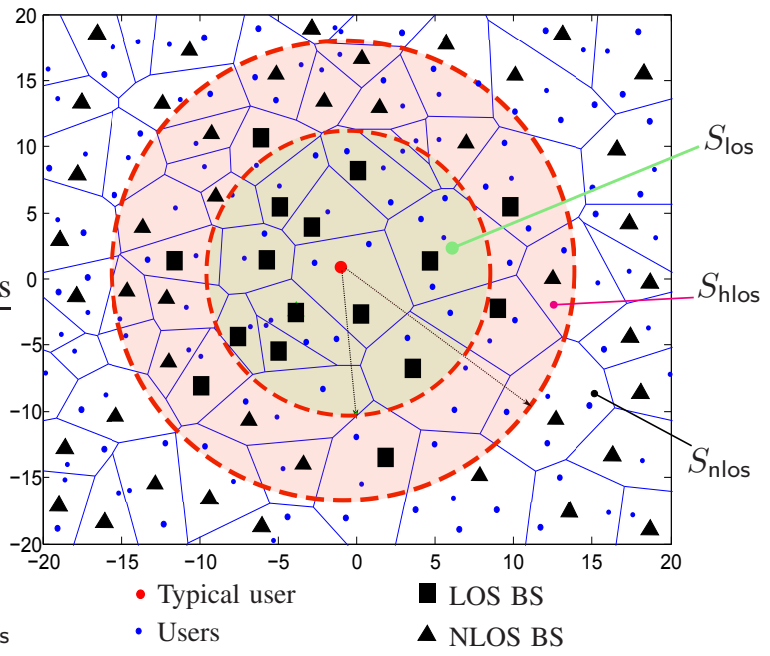

Fig. 1. $S_{\text {los }}$ and $S_{\text {nlos }}$ regions contain only LOS and NLOS BSs respectively, while $S_{\text {hlos }}$ contains a mixture of the two with probability $p(r)=1-$ $\frac{r-\mathrm{R}_{\text {los }}}{\mathrm{R}_{\mathrm{nlos}}-\mathrm{R}_{\text {los }}}$ for LOS BSs and $1-p(r)=\frac{r-\mathrm{R}_{\text {los }}}{\mathrm{R}_{\text {nlos }}-\mathrm{R}_{\text {los }}}$ for NLOS BSs.

where $K \triangleq \mathrm{R}_{\text {los }}^{\alpha_{\text {nlos }}-\alpha_{\text {los }}}$ is a parameter to ensure the continuity of the path-loss function as in [9].

For positive reals $m$ and $\mathrm{R}$, we consider the following pathloss functions of interest ${ }^{3}$

$$
\mathcal{L}_{1}\left(m ; r_{x}\right)=r_{x}^{-m} \text { and } \mathcal{L}_{2}\left(\mathrm{R} ; r_{x}\right)= \begin{cases}r_{x}^{-\alpha_{\text {los }}} & \text { if } r_{x} \leq \mathrm{R} \\ K r_{x}^{-\alpha_{\text {nlos }}} & \text { if } r_{x}>\mathrm{R} .\end{cases}
$$

\section{B. User Association Policy under the H-LOS probability model}

We consider the average power-based cell association policy. Since $\eta=\alpha_{\text {nlos }} / \alpha_{\text {los }} \geq 1$ and the $H$-LOS probability model is adopted, the strongest BS is the nearest one in the regions $S_{\text {los }}$ and $S_{\text {nlos }}$, while it is not necessarily the case in

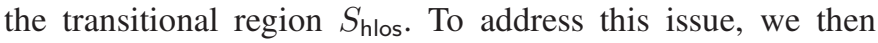
examine the distribution of distances between the typical user and the serving BS as a result of two events: the transmission link type (LOS or NLOS), and the region to which the serving BS belongs ( $S_{\text {los }}, S_{\text {nlos }}$ or $S_{\text {hlos }}$ ). For $i \in\{$ los, nlos $\}$, denote by $S_{1 i}$ the LOS and NLOS BSs of the $S_{\text {hlos }}$ region respectively, by $D_{i}$ the link distance from the typical user to $B_{i}$, the nearest BS of LOS and NLOS BSs respectively, and by $S$ the serving region, i.e., the region that contains the serving BS. For BSs in $\Phi_{i}$, the PDF of the horizontal distance $r_{x}$ is then expressed as

$$
f_{D_{i}}\left(r_{x}\right)=2 \pi \lambda r_{x} P_{i}\left(r_{x}\right) \exp \left(-2 \pi \lambda \int_{0}^{r_{x}} u P_{i}(u) \mathrm{d} u\right)
$$

Now given that $D_{i}=r_{x}$ and $B_{i}$ belongs to the $S_{\text {hlos }}$ region, it can be the serving BS if it verifies the following constraints:

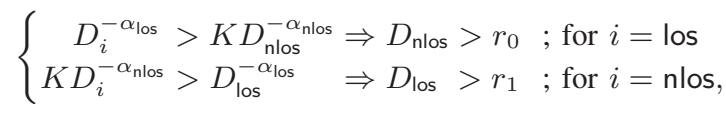

where $r_{0}=\mathrm{R}_{\text {los }}^{1-\frac{1}{\eta}} r_{x}^{\frac{1}{\eta}}$ and $r_{1}=\operatorname{Min}\left(\mathrm{R}_{\text {nlos }}, r_{x}^{\eta} / \mathrm{R}_{\text {los }}^{\eta-1}\right)$ holds since $S_{\text {nlos }}$ does not contain any LOS BS.

\footnotetext{
${ }^{3}$ Note that $\mathcal{L}(.) \equiv \mathcal{L}_{1}\left(\alpha_{\text {los }} ;.\right)$ when $\alpha_{\text {los }}=\alpha_{\text {nlos }}$, i.e., $\mathrm{R}_{\text {los }} \rightarrow \infty$ or $\mathrm{R}_{\text {nlos }} \rightarrow 0$, and $\mathcal{L}(.) \equiv \mathcal{L}_{2}\left(\mathrm{R}_{\text {los }} ;\right.$. $)$ when $\mathrm{R}_{\text {los }}=\mathrm{R}_{\text {nlos }}$.
} 
Given that $D_{i}=r_{x}$, the probability that the typical user will be connected to $B_{i}$ is then given by

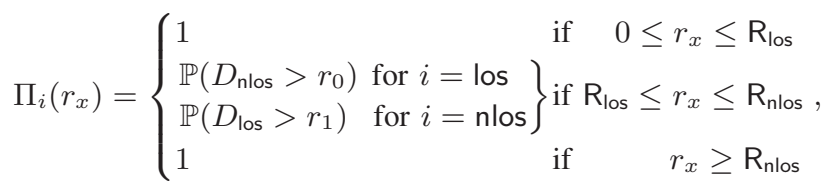

while $\mathbb{P}\left(D_{\text {nlos }}>r_{0}\right)$ and $\mathbb{P}\left(D_{\text {los }}>r_{1}\right)$ are computed using (16).

Remark 3. For $j \in \mathcal{J}=\{$ los, 1 los, 1 nlos, nlos $\}$, The association probability $\mathcal{A}_{j}=\mathbb{P}\left(S=S_{j}\right)$ that a typical user connects to a $B S$ from $S_{j}$, can be computed by integrating $\Pi_{i}\left(r_{x}\right) f_{D_{i}}\left(r_{x}\right)$ over each region radius interval. An interesting observation for the $S_{\text {los }}$ region, is that for fixed parameter $\mathrm{R}_{\text {los }}, \mathcal{A}_{\text {los }}=$ $1-\exp \left(-\pi \lambda \mathrm{R}_{\text {los }}^{2}\right)$ increases with $\lambda$, while the average number of users connected to $S_{\text {los }}$-expressed as $\widetilde{\mathcal{N}_{\text {los }}}=\left(\lambda_{u} / \lambda\right) \mathcal{A}_{\text {los }}$, where $\lambda_{u}$ is the density of the users PPP-decreases. However, for fixed $\lambda$, expanding $\mathrm{R}_{\text {los }}$ leads to an increase in $\mathcal{A}_{\text {los }}$ and $\overline{\mathcal{N}_{\text {los }}}$ simultaneously. More discussions are provided in Section V.

\section{Coverage Probability Analysis}

In this section, we analyze the coverage probability under our tractable system model provided in Section III, and aimed to capture the random impact of generalized shadowing and RNPO parameters via the fluctuation of aggregated parameters $\mathrm{R}_{\text {los }}$ and $\mathrm{R}_{\text {nlos }}$.

\section{A. Coverage Probability}

We define the coverage probability under the path-loss function defined in (14), as the probability $\mathcal{P}_{\mathcal{L}}^{\operatorname{SINR}}($.$) that the$ received SINR is greater than a threshold $\mathrm{T}$ when the serving BS belongs to one of the four sets $S_{\text {los }}, S_{1 \text { los }}, S_{1 \text { nlos }}$ or $S_{\text {nlos }}$.

Theorem 1. The coverage probability under the path-loss function (14) is given by

$$
\mathcal{P}_{\mathcal{L}}^{\text {SINR }}(.)=\mathcal{P}_{\text {los }}^{\text {SINR }}+\mathcal{P}_{1 \text { los }}^{\text {SINR }}+\mathcal{P}_{1 \text { los }}^{\text {SINR }}+\mathcal{P}_{\text {nlos }}^{\text {SINR }}
$$

where for $j \in \mathcal{J}=\{$ los, 1 los, 1 nlos, nlos $\}, \mathcal{P}_{j}^{\text {SINR }}$ stands for the coverage probability when the serving $B S$ belongs to $S_{j}$ and the supplementary equations are listed in the top of the next page such as $a=-1 /\left(\mathrm{R}_{\text {nlos }}-\mathrm{R}_{\text {los }}\right), b=-\mathrm{R}_{\text {nlos }} /\left(\mathrm{R}_{\text {nlos }}-\mathrm{R}_{\text {los }}\right), \rho_{m}=\left(\mathrm{R}_{\text {nlos }} / \mathrm{R}_{\text {los }}\right)^{m}$ for $m \in \mathbb{R}$, and $\delta_{p 0}=p / \alpha_{\text {los }}$ and $\delta_{p 1}=p / \alpha_{\text {nlos }}$ for $p=2$ or 3 .

Proof. The sketch of the proof is as follows: The coverage probability is expressed as $\mathcal{P}_{\mathcal{L}}^{\text {SINR }}()=.\sum_{j \in \mathcal{J}} \mathcal{P}_{j}^{\text {SINR }}()=$. $\sum_{j \in \mathcal{J}} \mathcal{A}_{j} \mathbb{P}\left(\operatorname{SINR}>\mathrm{T} \mid S=S_{j}\right)$, and each component of $\mathcal{P}_{\mathcal{L}}^{\text {SINR }}($.$) will be computed with the following similar steps$

$$
\begin{aligned}
& \mathcal{P}_{\text {los }}^{\text {SINR }}=\mathcal{A}_{\text {los }} \int_{0}^{R_{\text {los }}} \mathbb{P}\left(\operatorname{SINR}>\mathrm{T} \mid u, S=S_{\text {los }}\right) f_{D_{\text {los }}}\left(u \mid S=S_{\text {los }}\right) \mathrm{d} u \\
& \left.\stackrel{(\text { a) }}{=} \int_{0}^{\mathrm{R}_{\text {los }}} \mathbb{P}(\operatorname{SINR})>\mathrm{T} \mid u, S_{\text {los }}\right) \Pi_{\text {los }}(u) f_{D_{\text {los }}}(u) \mathrm{d} u
\end{aligned}
$$

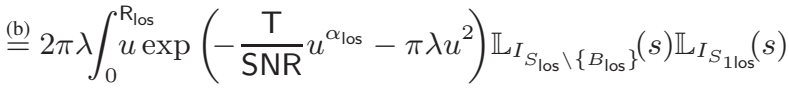

$$
\begin{aligned}
& \times \mathbb{L}_{I_{S_{1 \text { llos }}}}(s) \mathbb{L}_{I_{S_{\text {nlos }}}}(s) \mathrm{d} u,
\end{aligned}
$$

where $s=\mathrm{T} u^{\alpha_{\text {los }}}$. (a) follows from

$$
f_{D_{\text {los }}}\left(u \mid S=S_{\text {los }}\right)=\frac{\mathrm{d}}{\mathrm{d} u} \frac{\mathbb{P}\left(D_{\text {los }} \leq u, S=S_{\text {los }}\right)}{\mathbb{P}\left(S=S_{\text {los }}\right)}=\frac{\prod_{\text {los }}(u) f_{D_{\text {los }}}(u)}{\mathcal{A}_{\text {los }}} .
$$

(b) holds since $h_{B_{\text {los }}} \sim \exp (1)$ and the aggregated interference $I_{\text {agg }}=\sum_{x \in \Phi_{b}^{\prime} \backslash\left\{B_{\text {los }}\right\}} h_{x} \mathcal{L}\left(r_{x}\right)$ is seen as the summation of the interference power (normalized by $P_{\mathrm{tx}}$ ) from each set $U \in\left\{S_{\text {los }} \backslash\left\{B_{\text {los }}\right\}, S_{1 \text { los }}, S_{\text {1nlos }}, S_{\text {nlos }}\right\}$, i.e.,

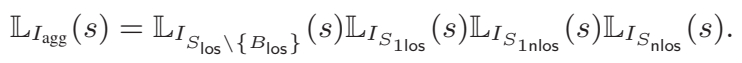

We get the desired result for $\mathcal{P}_{\text {los }}^{\text {SINR }}$ (.) in (19) by using the PGFL theorem to compute the Laplace transforms $\mathbb{L}_{I_{U}}($.$) and$ some variable changes.

Remark 4. Although the expression of coverage probability under the H-LOS model is in complicated form, it instigates an intuitive algorithmic development. Moreover, the expression is general enough to accommodate several previous expressions. For example, it reflects the 3GPP case 1 study in [14] when $\mathrm{R}_{\text {los }} \rightarrow 0$, and approximates the $3 G P P$ case 2 study when $\mathrm{R}_{\text {los }} \rightarrow \epsilon d_{1}$ and $\mathrm{R}_{\text {nlos }} \rightarrow d_{1} / \epsilon$ where $0<\epsilon<1$ is to adjust the approximation's error. More precisely, (18) generally approximates the coverage analysis under the models in [13] by simply adjusting the parameters $a$ and $b$. Furthermore, when $\mathrm{R}_{\text {nlos }} \simeq \mathrm{R}_{\text {los }}, \mathcal{L}$ becomes a dual-slope path-loss model $\mathcal{L}_{2}$ and (18) is simplified under the expression in [3, Th. 1]. If $\alpha_{\mathrm{nlos}} \simeq \alpha_{\mathrm{los}}$, (18) will be the same expression as [2, Th. 2].

\section{B. The H-LOS model and Ultra-Dense Networks}

We consider the scenario of ultra-dense networks [2], where the interference $I_{\mathrm{agg}}$ dominates the noise normalized by the transmit power $\left(\sigma^{2} / P_{\mathrm{tx}}\right)$. SINR is then approximated by $\mathrm{SIR}_{\mathcal{L}} \triangleq$ $\left.\operatorname{SINR}_{\mathcal{L}}\right|_{\frac{\sigma^{2}}{P_{\mathrm{tx}}}}=0$

Remark 5. In the interference-limited regime, the coverage probability in (18) remains invariant as long as $\lambda \mathrm{R}_{\text {los }}^{2}$ and $\lambda \mathrm{R}_{\mathrm{nlos}}^{2}$ are invariant. In other words, the impact on coverage probability of increasing/decreasing $\lambda$ is analogous to increasing/decreasing $\left(\mathrm{R}_{\mathrm{los}}, \mathrm{R}_{\mathrm{nlos}}\right)$ simultaneously, which is a generalization of [9, Fact 1].

In the following proposition, comparisons are made for $\mathcal{P}^{\mathrm{SIR}}$ under $\mathcal{L}_{1}, \mathcal{L}_{2}$ and $\mathcal{L}$.

Proposition 2. The following SIR coverage ordering holds for arbitrary $0<\alpha_{\text {los }} \leq \alpha_{\text {nlos }}$ and $\mathrm{R}_{\mathrm{los}} \leqslant \mathrm{R}_{\text {nlos }}$

(i) $\mathcal{P}_{\mathcal{L}(.)}^{\mathrm{SIR}}>\mathcal{P}_{\mathcal{L}_{2}\left(\mathrm{R}_{\text {nlos } ; .)}\right.}^{\mathrm{SIR}}>\mathcal{P}_{\mathcal{L}_{1}\left(\alpha_{\text {los } ; .)}\right.}^{\mathrm{SIR}}$.

(ii) $\mathcal{P}_{\mathcal{L}(.)}^{\mathrm{SIR}}<\mathcal{P}_{\mathcal{L}_{2}\left(\mathrm{R}_{\text {los } ; .)}\right.}^{\mathrm{SIR}}<\mathcal{P}_{\mathcal{L}_{1}\left(\alpha_{\text {nlos } ; .)}\right.}^{\mathrm{SIR}}$.

(iii) $\lim _{\lambda \rightarrow \infty} \mathcal{P}_{\mathcal{L}(.)}^{\mathrm{SINR}}=\lim _{\lambda \rightarrow \infty} \mathcal{P}_{\mathcal{L}(.)}^{\mathrm{SIR}}=\mathcal{P}_{\mathcal{L}_{1}\left(\alpha_{\text {los }} ; .\right)}^{\mathrm{SIR}}$.

(iv) $\lim _{\lambda \rightarrow \infty} \mathcal{P}_{\mathcal{L}(.)}^{\text {SINR }}=\lim _{\lambda \rightarrow \infty} \mathcal{P}_{\mathcal{L}(.)}^{\text {SIR }}()=$.0 when $\alpha_{\text {los }} \leq 2$.

(v) $\lim _{\lambda \rightarrow 0} \mathcal{P}_{\mathcal{L}(.)}^{\mathrm{SIR}}=\mathcal{P}_{\mathcal{L}_{1}\left(\alpha_{\text {nlos }} ; .\right)}^{\mathrm{SIR}}$.

Proof. The proof of (i) and (ii) is similar to that of [9, Lemma 2], the main change is to proceed by considering the two cases when the serving BS $x_{0} \in\left(S_{\text {los }} \cup S_{\text {nlos }}\right)$ (where $\mathcal{L} \equiv \mathcal{L}_{2}$ ) and $x_{0} \in S_{\text {hlos. }}$ (iii) and (v) follows from the observation of Remark 5 where $\lambda \rightarrow \infty \equiv\left(\mathrm{R}_{\text {los }}, \mathrm{R}_{\text {nlos }}\right) \rightarrow \infty$ and $\lambda \rightarrow 0 \equiv$ $\left(\mathrm{R}_{\text {los }}, \mathrm{R}_{\text {nlos }}\right) \rightarrow 0$. Such scaling in the definition of $\mathcal{L}($.$) results$ in $\mathcal{L}_{1}\left(\alpha_{\text {los }} ;\right.$. $)$ or $\mathcal{L}_{1}\left(\alpha_{\text {nlos }} ;\right.$. $)$. (iv) follows from combining (ii) and [9, Proposition 1]. While $\mathcal{P}_{\mathcal{L}(.)}^{\text {SINR }} \rightarrow \mathcal{P}_{\mathcal{L}(.)}^{\text {SIR }}$ as $\lambda \rightarrow \infty$ completes the proof. 


$$
\begin{aligned}
& \mathcal{P}_{\text {los }}^{\mathrm{SINR}}(\mathrm{T})=\pi \lambda \mathrm{R}_{\text {los }}^{2} \int_{0}^{1} \exp \left(-\frac{\mathrm{TR}_{\text {los }}^{\alpha_{\text {los }}}}{\mathrm{SNR}} x^{\frac{\alpha_{\text {los }}}{2}}-\pi \lambda \mathrm{R}_{\text {los }}^{2}\left[A_{\text {los }}^{(1)}(x)+\rho_{2} A_{\text {los }}^{(2)}(x)+\frac{2 a}{3} \mathrm{R}_{\text {los }} A_{\text {los }}^{(3)}(x)+b A_{\text {los }}^{(4)}(x)\right]\right) \mathrm{d} x \\
& \mathcal{P}_{1 \text { los }}^{\mathrm{SINR}}(\mathrm{T})=2 \pi \lambda \int_{\mathrm{R}_{\mathrm{los}}}^{\mathrm{R}_{\mathrm{nlos}}}\left(a r^{2}+b r\right) \exp \left(-\frac{\mathrm{T}}{\mathrm{SNR}} r^{\alpha_{\mathrm{los}}}-\pi \lambda\left[r_{0}^{2} A_{1 \text { los }}^{(1)}(r)+\mathrm{R}_{\mathrm{nlos}}^{2} A_{1 \text { los }}^{(2)}(r)+\frac{2 a}{3} A_{1 \text { los }}^{(3)}(r)+b A_{1 \text { los }}^{(4)}(r)\right]\right) \mathrm{d} r, \\
& \mathcal{P}_{\text {1nlos }}^{\text {SINR }}(\mathrm{T})=2 \pi \lambda \int_{\mathrm{R}_{\text {los }}}^{\mathrm{R}_{\text {nlos }}}\left([1-b] r-a r^{2}\right) \exp \left(\frac{-\mathrm{T} r^{\alpha_{\text {nlos }}}}{K \mathrm{SNR}}-\pi \lambda\left[r^{2} A_{\text {1nlos }}^{(1)}(r)+\mathrm{R}_{\text {nlos }}^{2} A_{\text {1nlos }}^{(2)}(r)+\frac{2 a}{3} A_{1 \text { nlos }}^{(3)}(r)+b A_{1 \text { nlos }}^{(4)}(r)\right]\right) \mathrm{d} r, \\
& \mathcal{P}_{\text {nlos }}^{\mathrm{SINR}}(\mathrm{T})=\pi \lambda \mathrm{R}_{\text {nlos }}^{2} \int_{1}^{\infty} \exp \left(-\frac{\mathrm{TR}_{\text {los }}^{\alpha_{\text {los }}} \rho_{\alpha_{\text {nlos }}}}{\mathrm{SNR}} x^{\frac{\alpha_{\text {nlos }}}{2}}-\pi \lambda \mathrm{R}_{\text {nlos }}^{2} x F_{-\delta_{21}}(\mathrm{~T})\right) \mathrm{d} x, \\
& A_{\text {los }}^{(1)}(x)=x\left[1-F_{\delta_{20}}\left(\frac{1}{\mathrm{~T}}\right)\right]+\rho_{2} F_{\delta_{21}}\left(\frac{\rho_{\alpha_{\mathrm{nlos}}}}{\mathrm{T} x^{\frac{1}{\delta_{20}}}}\right)+\left[F_{\delta_{20}}\left(\frac{1}{\mathrm{~T} x^{\frac{1}{\delta_{20}}}}\right)-F_{\delta_{21}}\left(\frac{1}{\mathrm{~T} x^{\frac{1}{\delta_{20}}}}\right)\right], A_{\text {los }}^{(2)}(x)=F_{-\delta_{21}}\left(\frac{\mathrm{T}}{\rho_{\alpha_{\text {nlos }}}} x^{\frac{1}{\delta_{20}}}\right)-1 \text {, } \\
& A_{\text {los }}^{(3)}(x)=\rho_{3}\left[F_{\delta_{30}}\left(\frac{\rho_{\alpha_{\text {los }}}}{\mathrm{T} x^{\frac{1}{\delta_{20}}}}\right)-F_{\delta_{31}}\left(\frac{\rho_{\alpha_{\text {nlos }}}}{\mathrm{T} x^{\frac{1}{\delta_{20}}}}\right)\right]-\left[F_{\delta_{30}}\left(\frac{1}{\mathrm{~T}^{\frac{1}{\delta_{20}}}}\right)-F_{\delta_{31}}\left(\frac{1}{\mathrm{~T} x^{\frac{1}{\delta_{20}}}}\right)\right] \text {, } \\
& A_{\text {los }}^{(4)}(x)=\rho_{2}\left[F_{\delta_{20}}\left(\frac{\rho_{\alpha_{\text {los }}}}{\mathrm{T} x^{\frac{1}{\delta 20}}}\right)-F_{\delta_{21}}\left(\frac{\rho_{\alpha_{\text {nlos }}}}{\mathrm{T}^{\frac{1}{\delta_{20}}}}\right)\right]-\left[F_{\delta_{20}}\left(\frac{1}{\mathrm{~T}^{\frac{1}{\delta_{20}}}}\right)-F_{\delta_{21}}\left(\frac{1}{\mathrm{~T} x^{\frac{1}{\delta_{20}}}}\right)\right] \text {, } \\
& A_{1 \mathrm{los}}^{(1)}(r)=1-F_{\delta_{21}}\left(\frac{1}{\mathrm{~T}}\right), \quad A_{1 \mathrm{los}}^{(2)}(r)=F_{\delta_{21}}\left(\frac{\rho_{\alpha_{\mathrm{nlos}}} \mathrm{R}_{\mathrm{los}}^{\alpha_{\mathrm{los}}}}{\mathrm{T} r^{\alpha_{\mathrm{los}}}}\right)+F_{-\delta_{21}}\left(\frac{\mathrm{T} r^{\alpha_{\mathrm{los}}}}{\rho_{\alpha_{\mathrm{nlos}}} \mathrm{R}_{\mathrm{los}}^{\alpha_{\mathrm{los}}}}\right)-1, \\
& A_{1 \mathrm{los}}^{(3)}(r)=r_{0}^{3}\left[F_{\delta_{31}}\left(\frac{1}{\mathrm{~T}}\right)-1\right]-r^{3}\left[F_{\delta_{30}}\left(\frac{1}{\mathrm{~T}}\right)-1\right]+\mathrm{R}_{\mathrm{nlos}}^{3}\left[F_{\delta_{30}}\left(\frac{\mathrm{R}_{\mathrm{nlos}}^{\alpha_{\mathrm{los}}}}{\mathrm{T} r^{\alpha_{\mathrm{los}}}}\right)-F_{\delta_{31}}\left(\frac{\rho_{\alpha_{\text {nlos }}} \mathrm{R}_{\mathrm{los}}^{\alpha_{\mathrm{los}}}}{\mathrm{T} r^{\alpha_{\mathrm{los}}}}\right)\right] \text {, } \\
& A_{1 \mathrm{los}}^{(4)}(r)=r_{0}^{2}\left[F_{\delta_{21}}\left(\frac{1}{\mathrm{~T}}\right)-1\right]-r^{2}\left[F_{\delta_{20}}\left(\frac{1}{\mathrm{~T}}\right)-1\right]+\mathrm{R}_{\mathrm{nlos}}^{2}\left[F_{\delta_{20}}\left(\frac{\mathrm{R}_{\mathrm{nlos}}^{\alpha_{\mathrm{los}}}}{\mathrm{T} r^{\alpha_{\mathrm{los}}}}\right)-F_{\delta_{21}}\left(\frac{\rho_{\alpha_{\mathrm{nlos}}} \mathrm{R}_{\mathrm{los}}^{\alpha_{\mathrm{los}}}}{\mathrm{T} r^{\alpha_{\mathrm{los}}}}\right)\right] \text {, } \\
& A_{1 \mathrm{nlos}}^{(1)}(r)=1-F_{\delta_{21}}\left(\frac{1}{\mathrm{~T}}\right), A_{1 \mathrm{nlos}}^{(2)}(r)=F_{\delta_{21}}\left(\frac{\mathrm{R}_{\mathrm{nlos}}^{\alpha_{\mathrm{nlos}}}}{\mathrm{T} r^{\alpha_{\mathrm{nlos}}}}\right)+F_{-\delta_{21}}\left(\frac{\mathrm{T}^{\alpha_{\mathrm{nlos}}}}{\mathrm{R}_{\mathrm{nlos}}^{\alpha_{\mathrm{nlos}}}}\right)-1, \\
& A_{1 \mathrm{nlos}}^{(3)}(r)=r^{3}\left[F_{\delta_{31}}\left(\frac{1}{\mathrm{~T}}\right)-1\right]-r_{1}^{3}\left[F_{\delta_{30}}\left(\frac{K r_{1}^{\alpha_{\mathrm{los}}}}{\mathrm{T} r^{\alpha_{\mathrm{nlos}}}}\right)-1\right]+\mathrm{R}_{\mathrm{nlos}}^{3}\left[F_{\delta_{30}}\left(\frac{\rho_{\alpha_{\mathrm{los}}} \mathrm{R}_{\text {los }}^{\alpha_{\mathrm{nlos}}}}{\mathrm{T} r^{\alpha_{\mathrm{nlos}}}}\right)-F_{\delta_{31}}\left(\frac{\mathrm{R}_{\mathrm{nlos}}^{\alpha_{\mathrm{nlos}}}}{\mathrm{T} r^{\alpha_{\mathrm{nlos}}}}\right)\right] \text {, } \\
& A_{1 \mathrm{nlos}}^{(4)}(r)=r^{2}\left[F_{\delta_{21}}\left(\frac{1}{\mathrm{~T}}\right)-1\right]-r_{1}^{2}\left[F_{\delta_{20}}\left(\frac{K r_{1}^{\alpha_{\mathrm{los}}}}{\mathrm{T} r^{\alpha_{\mathrm{nlos}}}}\right)-1\right]+\mathrm{R}_{\mathrm{nlos}}^{2}\left[F_{\delta_{20}}\left(\frac{\rho_{\alpha_{\mathrm{los}}} \mathrm{R}_{\text {los }}^{\alpha_{\mathrm{nlos}}}}{\mathrm{T} r^{\alpha_{\mathrm{nlos}}}}\right)-F_{\delta_{21}}\left(\frac{\mathrm{R}_{\mathrm{nlos}}^{\alpha_{\text {nlos }}}}{\mathrm{T} r^{\alpha_{\mathrm{nlos}}}}\right)\right] \text {. }
\end{aligned}
$$

\section{The Regime of Optimal BS Density}

We define the optimal BS density $\lambda_{\mathcal{L}}^{\text {opt }}$ as the specific $\lambda$ that maximizes the coverage probability under the path-loss function $\mathcal{L}$. Mathematically,

$$
\lambda_{\mathcal{L}}^{\mathrm{opt}}(.)=\underset{\lambda}{\arg }\left[\frac{\partial \mathcal{P}_{\mathcal{L}}^{\mathrm{SINR}}(.)}{\partial \lambda}=0\right] .
$$

Using a combination of proposition 2 and [9, lemma 4], $\mathcal{P}_{\mathcal{L}}^{\mathrm{SINR}}(\lambda)$ is a decreasing function when $\lambda>\lambda_{\mathcal{L}}^{\mathrm{opt}}$ and SINR $\simeq$ SIR. $\lambda_{\mathcal{L}}^{\text {opt }}$ can then be seen as the BS density to enter the SIR regime. We define the optimal regime under $\mathcal{L}$, the regime where the $\mathrm{BS}$ density $\lambda \simeq \lambda_{\mathcal{L}}^{\text {opt }}$. In this regime, the noise normalized by the transmit power is small w.r.t. the aggregated interference but it is non-zero. Consequently, (i)(ii) of proposition 2 are at first stages to be met. We have then

$$
\begin{aligned}
& \mathcal{P}_{\mathcal{L}_{1}\left(\alpha_{\text {los }} ; .\right)}^{\text {SINR }}<\mathcal{P}_{\mathcal{L}(.)}^{\text {SINR }}<\mathcal{P}_{\mathcal{L}_{1}\left(\alpha_{\text {nlos }} ; .\right)}^{\text {SIINR }} . \\
& \lambda_{\mathcal{L}_{2}\left(\mathrm{R}_{\text {nlos } ; .)}\right.}^{\text {opt }}<\lambda_{\mathcal{L}}^{\text {opt }}<\lambda_{\mathcal{L}_{2}\left(\mathrm{R}_{\text {los }} ; .\right)}^{\text {opt }} .
\end{aligned}
$$

Due to the lack of general closed-form expression for $\mathcal{P}_{\mathcal{L}_{1}(\alpha ; .)}^{S \text {. }}$ that would avoid the computation of a two-fold numerical integral in [1, theorem 1], almost all literature works focus on the $\mathcal{Q}$-function based expression when the path-loss exponent $\alpha=4$, which is only typical for terrestrial propagation at moderate to large distances. The following proposition overcome this limitation by developing closed-form expressions for
$\mathcal{P}_{\mathcal{L}_{1}(\alpha ; \text {.) }}^{\text {SINR }}$ considering all integer $\alpha>2$ (not only $\alpha=4$ ) and then conclude closed-form bounds for $\mathcal{P}_{\mathcal{L}(.)}^{\text {SINR }}$ in the optimal regime.

Proposition 3. For integer path-loss exponents $\alpha_{\text {los }}$ and $\alpha_{\text {nlos }}$ such as $2<\alpha_{\text {los }}<\alpha_{\text {nlos. }} \mathcal{P}_{\mathcal{L}}^{\text {SINR }}$ is bounded in the optimal regime as follows $\mathcal{P}_{\mathcal{L}_{1}\left(\alpha_{\text {los }} ; .\right)}^{\text {SINR }}<\mathcal{P}_{\mathcal{L}(.)}^{\text {SINR }}<\mathcal{P}_{\mathcal{L}_{1}\left(\alpha_{\text {nlos }} ; .\right)}^{\text {SINR }}$ such as the lower and upper bounds are achievable by respectively increasing $\mathrm{R}_{\mathrm{los}}$ and decreasing $\mathrm{R}_{\mathrm{nlos}}$, and where for even and odd values of $\alpha$, respectively

$$
\begin{aligned}
& \mathcal{P}_{\mathcal{L}_{1}(\alpha ; .)}^{\text {SINR }}=\frac{2 \pi \lambda}{\alpha(\mathrm{T} / \mathrm{SNR})^{\frac{2}{\alpha}}} \sum_{k=0}^{\frac{\alpha}{2}-1} \frac{(-1)^{k} \kappa^{k}}{k !} \Gamma\left(\frac{2+2 k}{\alpha}\right) \\
& \times{ }_{1} F_{\frac{\alpha-2}{2}}\left(\frac{4+2 k}{\alpha}, \ldots, \frac{\alpha+2 k}{\alpha} \mid \frac{(-\kappa)^{\frac{\alpha}{2}}}{\left(\frac{\alpha}{2}\right)^{\frac{\alpha}{2}}}\right), \\
& \mathcal{P}_{\mathcal{L}_{1}(\alpha ; .)}^{\mathrm{SINR}}=\frac{2 \pi \lambda}{\alpha(\mathrm{T} / \mathrm{SNR})^{\frac{2}{\alpha}}} \sum_{k=0}^{\alpha-1} \frac{(-1)^{k} \kappa^{k}}{k !} \Gamma\left(\frac{2+2 k}{\alpha}\right) \\
& \times{ }_{2} F_{\alpha-1}\left(\begin{array}{c}
1, \frac{1}{2}+\frac{k+1}{\alpha} \\
\left.\frac{2+k}{\alpha}, \ldots, \frac{\alpha+k}{\alpha} \mid \frac{4(-\kappa)^{\alpha}}{\alpha^{\alpha}}\right),
\end{array}\right.
\end{aligned}
$$

such as $\kappa=\frac{\pi \lambda F_{-\delta}(\mathrm{T})}{(\mathrm{T} / \mathrm{SNR})^{\delta}}, \delta=\frac{2}{\alpha}, \Gamma($.$) is the complete$ Gamma function and ${ }_{p} F_{q}($.$) is the generalized hypergeometric$ function.

Proof. By the variable change $(T / \mathrm{SNR}) x^{\alpha / 2} \rightarrow x$, the expression of $\mathcal{P}_{\mathcal{L}_{1}(\alpha ; .)}^{\text {SINR }}$ in [1, Theorem 2] can be rewritten as 


$$
\begin{aligned}
\mathcal{P}_{\mathcal{L}_{1}(\alpha ; .)}^{\mathrm{SINR}} & =\frac{2 \pi \lambda}{\alpha(\mathrm{T} / \mathrm{SNR})^{\frac{2}{\alpha}}} \int_{0}^{\infty} x^{\frac{2}{\alpha}-1} e^{-x} e^{-\kappa x^{2 / \alpha}} \mathrm{d} x \\
& =\frac{2 \pi \lambda}{\alpha(\mathrm{T} / \mathrm{SNR})^{\frac{2}{\alpha}}} \int_{0}^{\infty} x^{\frac{2}{\alpha}-1} e^{-x}{ }_{0} F_{0}\left(. ; . ;-\kappa x^{2 / \alpha}\right) \mathrm{d} x .
\end{aligned}
$$

Depending on the parity of $\alpha$, we use [15, Eq. (43)] (with $\alpha / 2$ order for the even case and $\alpha$ order for the odd one). Next, we explore the integral transformation of hypergeometric functions in $[16,(1.7 .525)]$. The proof is completed by combining (24) with Remark 5.

Using Proposition 3, the $\mathcal{Q}$-function based expression for $\alpha=4$ in [1], can be rewritten for $\kappa=\pi \lambda F_{-0.5}(\mathrm{~T}) \sqrt{\mathrm{SNR} / \mathrm{T}}$ as

$$
\begin{aligned}
\mathcal{P}_{\mathcal{L}_{1}(4 ; .)}^{\text {SINR }} & =\frac{\pi^{\frac{3}{2}} \lambda}{2 \sqrt{\mathrm{T} / \mathrm{SNR}}}\left[0 F_{0}\left(-;-\mid \frac{\kappa^{2}}{4}\right)-\frac{\kappa}{\sqrt{\pi}}{ }_{1} F_{1}\left(1 ; \frac{3}{2} \mid \frac{\kappa^{2}}{4}\right)\right] \\
& =\frac{\pi^{\frac{3}{2}} \lambda}{\sqrt{\mathrm{T} / \mathrm{SNR}}} \mathcal{Q}\left(\frac{\kappa}{\sqrt{2}}\right) \exp \left(\frac{\kappa^{2}}{4}\right) .
\end{aligned}
$$

While proposition 3 gives a complete characterization of $\mathcal{P}_{\mathcal{L}}$ in the optimal regime. The following proposition gives the scaling law of $\lambda_{\mathcal{L}}^{\text {opt }}$ as $\mathrm{R}_{\text {los }} \rightarrow \infty$ and $\mathrm{R}_{\text {nlos }} \rightarrow 0$.

Proposition 4. Under the H-LOS probability model such as $2<\alpha_{\text {los }}<\alpha_{\text {nlos }}$, the optimal BS density scales as follows

$$
\begin{aligned}
& \text { (i) } \lambda_{\mathcal{L}}^{\text {opt }}=\Omega\left(\left(\frac{\mathrm{T}}{\mathrm{SNR}}\right)^{\delta_{20}} \frac{1}{\pi F_{-\delta_{20}}(\mathrm{~T})}\right) \text { if } \mathrm{R}_{\mathrm{los}} \rightarrow \infty . \\
& \text { (ii) } \lambda_{\mathcal{L}}^{\mathrm{opt}}=O\left(\left(\frac{\mathrm{T}}{\mathrm{SNR}}\right)^{\delta_{21}} \frac{1}{\pi F_{-\delta_{21}}(\mathrm{~T})}\right) \text { if } \mathrm{R}_{\mathrm{nlos}} \rightarrow 0 .
\end{aligned}
$$

Proof. Using [9, Theorem 1], $\mathcal{P}_{\mathcal{L}_{2}\left(R_{c} ; .\right)}^{\text {SIIR }}$ is expressed for a given radius $\mathrm{R}_{c}$ as

$$
\mathcal{P}_{\mathcal{L}_{2}}^{\mathrm{SINR}}=\underbrace{\lambda \pi R_{c}^{2} \int_{0}^{1} \mathrm{e}^{-I_{f}(x)-W_{f}(x)} \mathrm{d} x}_{f(.)}+\underbrace{\lambda \pi R_{c}^{2} \int_{1}^{\infty} \mathrm{e}^{-I_{g}(x)-W_{g}(x)} \mathrm{d} x}_{g(.)},
$$

where $I_{f}(x)=\lambda \pi R_{c}^{2}\left(F_{\delta_{20}}\left(\frac{1}{\mathrm{~T} x^{\frac{1}{\delta_{20}}}}\right)+F_{-\delta_{20}}\left(\mathrm{~T} x^{\frac{1}{\delta_{20}}}\right)\right)$

$$
+\lambda \pi R_{c}^{2} x\left(1-F_{\delta_{20}}\left(\frac{1}{\mathrm{~T}}\right)\right)-\lambda \pi R_{c}^{2}, W_{f}(x)=\frac{\mathrm{T}}{\mathrm{SNR}} R_{c}^{\alpha_{\mathrm{los}}} x^{\frac{\alpha_{105}}{2}},
$$

$I_{g}(x)=\pi \lambda R_{c}^{2} x F_{-\delta_{21}}(\mathrm{~T})$ and $W_{g}(x)=\frac{\mathrm{T}}{\mathrm{SNR}} R_{c}^{\alpha_{\text {los }}} x^{\frac{\alpha_{\text {nlos }}}{2}}$.

We note that $I_{f}$ and $I_{g}$ are the terms reflecting interference while $W_{f}$ and $W_{g}$ are those capturing noise. In the optimal regime under $\mathcal{L}_{2}$, i.e., $\lambda \simeq \lambda_{\mathcal{L}_{2}\left(\mathrm{R}_{c}: \text {.) }\right.}^{\mathrm{opt}}, W_{f}$ and $W_{g}$ are respectively negligible w.r.t. $I_{f}$ and $I_{g}$ but non zero. We expand then the terms $\mathrm{e}^{-W_{f}(x)}$ and $\mathrm{e}^{-W_{g}(x)}$ as $\mathrm{e}^{-\mu}=$ $\sum_{k=0}^{n} \frac{(-\mu)^{k}}{k !}+E_{n}(\mu)$, where $E_{n}$ is the error of approximation such as $E_{n}(\mu) \leq \frac{|\mu|^{n+1}}{(n+1) !}$ [17]. The error of approximation of $\mathcal{P}_{\mathcal{L}_{2}}^{\text {SINR }}$ in the optimal regime is then upper bounded as

$$
\begin{aligned}
& \left|E_{n}\right| \leq \lambda \pi R_{c}^{2} A^{n+1} U_{n}+\lambda \pi R_{c}^{2} B^{n+1} V_{n}, \text { where } \\
& A \simeq \frac{\mathrm{T}}{\left(\lambda \pi F_{-\delta_{20}}(\mathrm{~T})\right)^{\frac{\alpha_{\text {los }}}{2}} \mathrm{SNR}}, B=\frac{\mathrm{T} R_{c}^{\alpha_{\text {los }}}}{\left(\lambda \pi R_{c}^{2} F_{-\delta_{21}}(\mathrm{~T})\right)^{\frac{\alpha_{\text {nlos }}}{2} \mathrm{SNR}},} \\
& U_{n}=\frac{\gamma\left((n+1) \frac{\alpha_{\text {los }}}{2}+1, \pi \lambda R_{c}^{2} F_{-\delta_{20}}(\mathrm{~T})\right)}{(n+1) !}, \text { and } \\
& V_{n}=\frac{\Gamma\left((n+1) \frac{\alpha_{\text {nlos }}}{2}+1, \pi \lambda R_{c}^{2} F_{-\delta_{21}}(\mathrm{~T})\right)}{(n+1) !},
\end{aligned}
$$

where $\gamma(s, x)=\int_{0}^{x} t^{s-1} \mathrm{e}^{-t} \mathrm{~d} t$ and $\Gamma(s, x)=\int_{x}^{\infty} t^{s-1} \mathrm{e}^{-t} \mathrm{~d} t$ are the lower and upper incomplete gamma function.

For any given error tolerance $\epsilon$, the bound (28) gives

$$
A \leq\left(\frac{\epsilon}{\lambda \pi R_{c}^{2}} \frac{1}{2 U_{n}}\right)^{\frac{1}{n+1}}, \quad B \leq\left(\frac{\epsilon}{\lambda \pi R_{c}^{2}} \frac{1}{2 V_{n}}\right)^{\frac{1}{n+1}} .
$$

If $R_{c} \rightarrow \infty$ and since $\alpha_{\mathrm{los}}>2, U_{n} \rightarrow \infty$ as $n \rightarrow \infty$ and then $\left(\frac{\epsilon}{\lambda \pi R_{c}^{2}} \frac{1}{2 U_{n}}\right)^{\frac{1}{n+1}} \rightarrow 1$ as $n \rightarrow \infty$. (29).1 simplifies as

$$
\begin{aligned}
\lambda & \geq\left(\frac{\mathrm{T}}{\mathrm{SNR}}\right)^{\delta_{20}} \frac{1}{\pi F_{-\delta_{20}}(\mathrm{~T})}, \\
\Rightarrow \exists \omega_{f} & \geq 1 \text { such as } \lambda_{\mathcal{L}_{2}\left(\mathrm{R}_{c} ; .\right)}^{\mathrm{opt}}=\left(\frac{\mathrm{T}}{\mathrm{SNR}}\right)^{\delta_{20}} \frac{\omega_{f}}{\pi F_{-\delta_{20}}(\mathrm{~T})} .
\end{aligned}
$$

If $R_{c} \rightarrow 0$ and since $\alpha_{\text {nlos }}>2, V_{n} \rightarrow \infty$ as $n \rightarrow \infty$ and then $\left(\frac{\epsilon}{\lambda \pi R_{c}^{2}} \frac{1}{2 V_{n}}\right)^{\frac{1}{n+1}} \rightarrow 1$ as $n \rightarrow \infty$. (29).2 simplifies as

$$
\begin{aligned}
\lambda & \geq\left(\frac{\mathrm{T}}{\mathrm{SNR}}\right)^{\delta_{21}} \frac{1}{\pi F_{-\delta_{21}}(\mathrm{~T})}, \\
\Rightarrow \exists \omega_{g} & \geq 1 \text { such as } \lambda_{\mathcal{L}_{2}\left(\mathrm{R}_{c} ; .\right)}^{\text {opt }}=\left(\frac{\mathrm{T}}{\mathrm{SNR}}\right)^{\delta_{21}} \frac{\omega_{g}}{\pi F_{-\delta_{21}}(\mathrm{~T})} .
\end{aligned}
$$

The proof is completed by combining (31) and (33) with (25).

Remark 6. By varying one parameter and fixing the others in (26) and (27), $\lambda_{\mathcal{L}}^{\text {opt }}$ is monotonically increasing with the SINR target $\mathrm{T}$, the noise variance $\sigma^{2}$ and the path-loss exponents, while it is decreasing with the transmit power $P_{t x}$ (intuitively, the higher you increase $P_{t x}$ the less you will need more BSs). Besides, $\lambda_{\mathcal{L}}^{\text {opt }}$ cannot be increased indefinitely with $\mathrm{T}$. In fact, for a real $0<m<1, \psi_{m}: \mathrm{T} \rightarrow \mathrm{T}^{m} / F_{-m}(\mathrm{~T})$ is an increasing function bounded as $\psi_{m}(\mathbf{T}) \leq \lim _{\mathbf{T} \rightarrow \infty} \psi_{m}(\mathbf{T})=\frac{1}{\varphi(m)}$, where $\varphi(m)=\int_{0}^{\infty} \frac{d u}{1+u^{\frac{1}{m}}}$ is finite (Riemann integral).

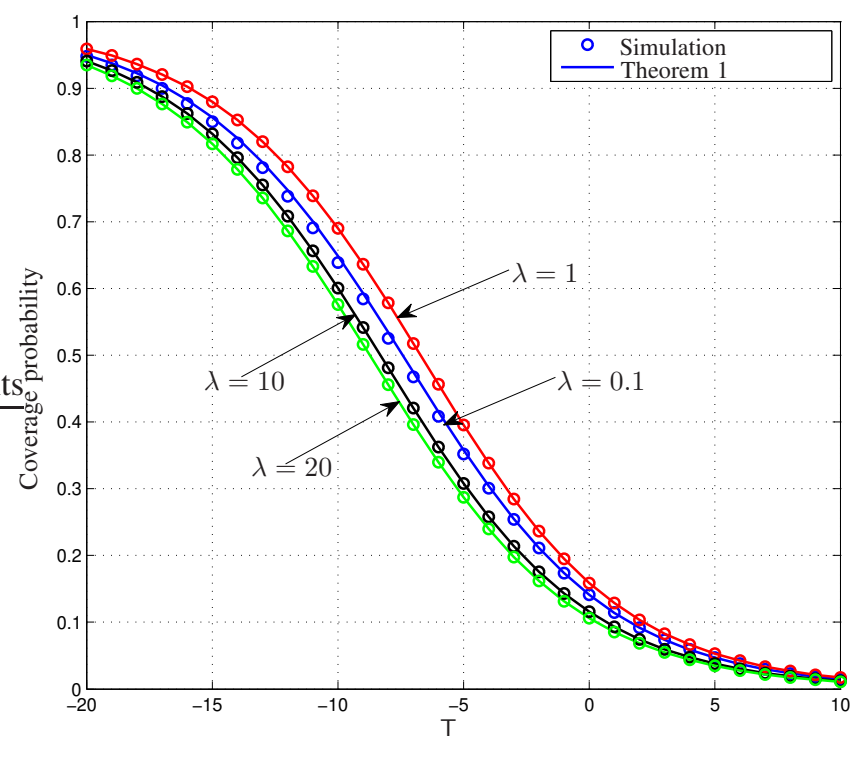

Fig. 2. Coverage probability from both Theorem 1 and simulation results. $\alpha_{\mathrm{los}}=2, \alpha_{\mathrm{nlos}}=4, \mathrm{R}_{\mathrm{los}}=1 \mathrm{~m}$ and $\mathrm{R}_{\mathrm{nlos}}=10 \mathrm{~m}$. 


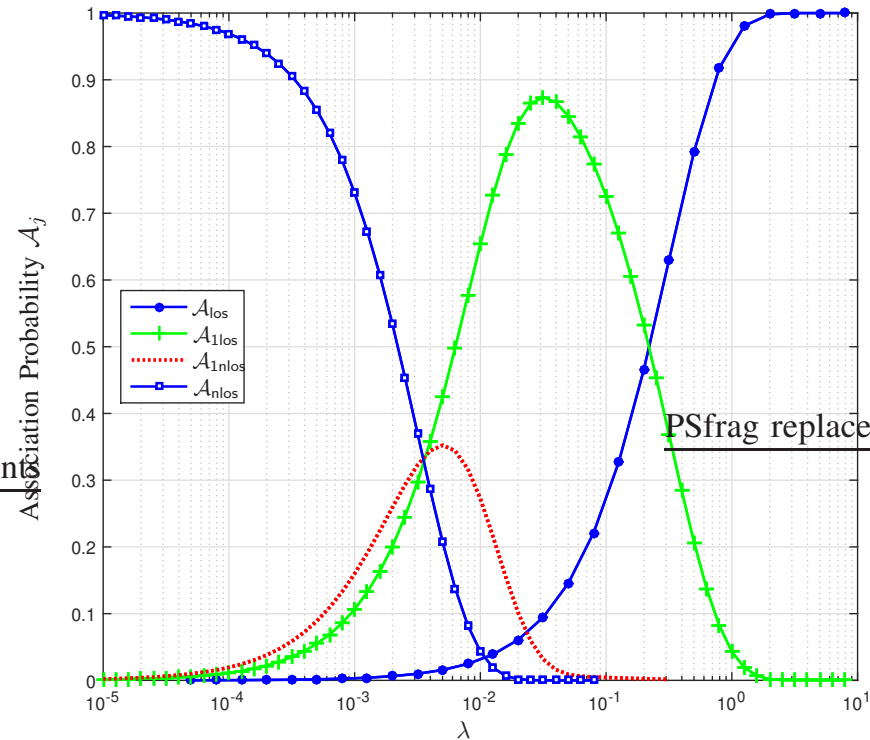

Fig. 3. Association probability scaling with BS density $\lambda$ for $\mathrm{R}_{\text {los }}=1 \mathrm{~m}$ and $\mathrm{R}_{\mathrm{nlos}}=10 \mathrm{~m}$

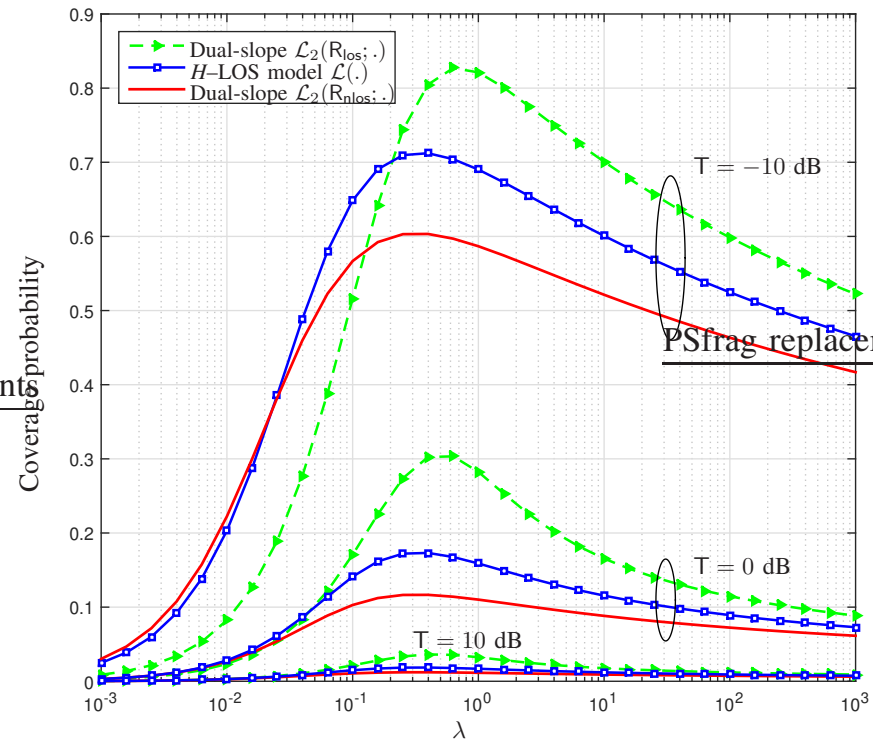

Fig. 4. Coverage probability scaling with $\lambda$ under $\mathcal{L}$ and $\mathcal{L}_{2}$ path-loss models, $\mathrm{R}_{\text {los }}=1 \mathrm{~m}$ and $\mathrm{R}_{\text {nlos }}=10 \mathrm{~m}, \alpha_{\text {los }}=2$ and $\alpha_{\text {nlos }}=4$.

\section{NumericAl Results AND DISCUSSIONS}

In this section, we present numerical results to assess our theoretical analysis. In the following, SNR $=0 \mathrm{~dB}$, integral expressions are evaluated using Matlab and Monte carlo simulations are performed with $10^{6}$ iterations.

\section{A. Validation of the model}

The expression of coverage probability in (18) configured with path-loss exponents $\alpha_{\text {los }}=2, \alpha_{\text {nlos }}=4$ and a given realization of BSs, shadowing and RNPO parameters such as $R_{\text {los }}=1 \mathrm{~m}$ and $R_{\text {nlos }}=10 \mathrm{~m}$, is plotted in Fig. 2. The

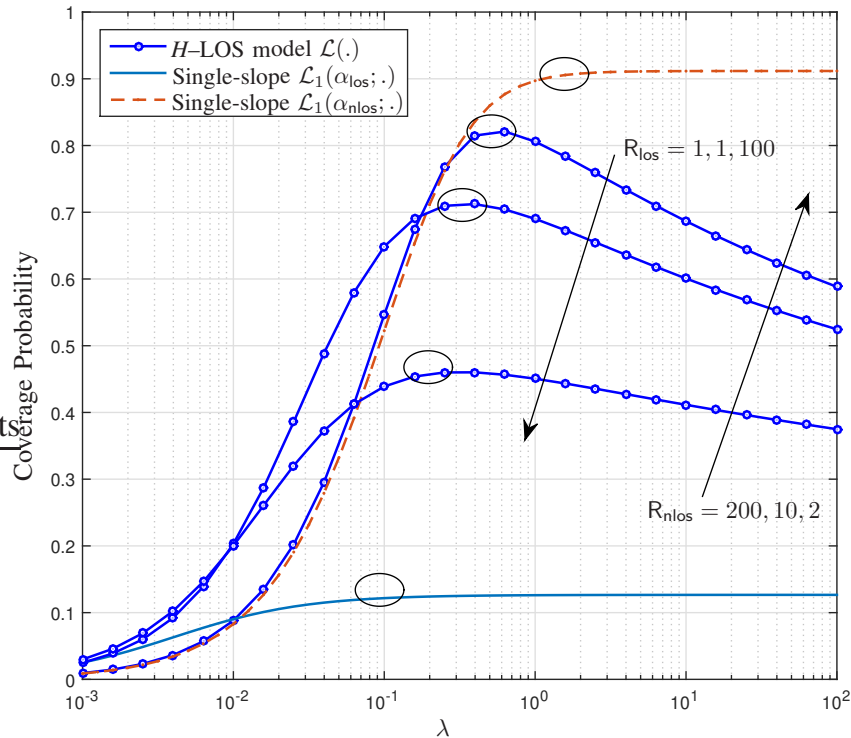

Fig. 5. Scaling of $\lambda_{\mathcal{L}}^{\mathrm{opt}}$ with $\mathrm{R}_{\text {los }}$ and $\mathrm{R}_{\text {nlos }}$ variations, $\alpha_{\text {los }}=2.03, \alpha_{\text {nlos }}=4$ and $\mathrm{T}=-10 \mathrm{~dB}$.

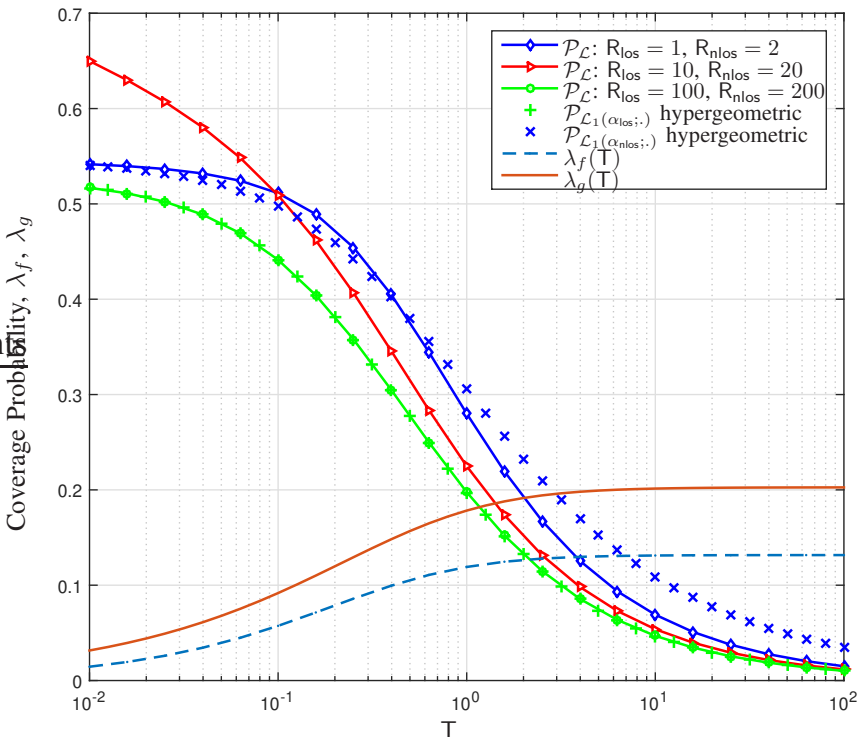

Fig. 6. Coverage probability scaling with network density $\lambda$ for $\mathcal{L}$ and $\mathcal{L}_{2}$ path-loss models. $\mathrm{R}_{\text {los }}=1 \mathrm{~m}$ and $\mathrm{R}_{\text {nlos }}=10 \mathrm{~m}$ and path-loss exponents $\alpha_{\text {los }}=2$ and $\alpha_{\text {nlos }}=4$.

plots show that the analytical expression match the simulation results well, and hence the accuracy of our theoretical analysis is validated. In particular, Fig. 2 shows that the coverage probability increases at first with network density $\lambda$ until achieving the optimal value $\lambda_{\mathcal{L}}^{\text {opt }}$, after that $\mathcal{P}_{\mathcal{L}}^{\text {SINR }}$ shrinks down as densification continue.

\section{B. The Association probabilities and operational regimes}

A combination of Fig. 3 and Fig. 4, reveals that when $\lambda<$ $0.0035 \mathrm{BSs} / \mathrm{m}^{2}$, the serving BS is potentially to be a BS from the $S_{\text {nlos }}$ set and the operational regime is the noise-limited 
regime where $I_{\text {agg }} \ll\left(\sigma^{2} / P_{\mathrm{tx}}\right)$; this is due to the observation that the network will be more sparse and the inter-distance between BSs is high enough such that $I_{\text {agg }}$ can be ignored. As $\lambda$ slightly increases $\left(\lambda \rightarrow 0.0035 \mathrm{BSs} / \mathrm{m}^{2}\right)$, the typical user is more likely to connect unsteadily to an NLOS BS from the hybrid region $S_{\text {hlos }}$. By continuously adding more BSs $\left(0.0035 \mathrm{BSs} / \mathrm{m}^{2}<\lambda<0.2 \mathrm{BSs} / \mathrm{m}^{2}\right)$, the serving BS crosses to be a LOS BS from $S_{\text {hlos }}$. Once $\lambda$ is large enough $\left(\lambda>0.2 \mathrm{BSs} / \mathrm{m}^{2}\right)$, the typical user is most likely to connect to a BS from $S_{\text {los }}$ and thus the coverage probability continues to increase until $\lambda$ achieves a specific value $\lambda_{\mathcal{L}}^{\mathrm{opt}} \simeq 0.4 \mathrm{BSs} / \mathrm{m}^{2}$. At that level, $\mathcal{P}_{\mathcal{L}_{3}}^{\text {SIR }}$ achieves its maximum value and follows the regression driven by interference $I_{\text {agg }}$ as $\lambda$ continue to increase.

\section{Coverage Probability and BS Density Scaling in The Optimal Regime}

Fig. 4 and Fig. 5 verifies Proposition 2 in the optimal regime as the coverage probability $\mathcal{P}_{\mathcal{L}}^{\text {SINR }}$ and the optimal BS density $\lambda_{\mathcal{L}}^{\text {opt }}$ remain bounded between those achieved under the standard and dual-slope path-loss functions. Numerically, $0.12<\mathcal{P}_{\mathcal{L}}^{\text {SINR }}<0.9$ and $0.1 \mathrm{BSs} / \mathrm{m}^{2}<\lambda_{\mathcal{L}}^{\text {opt }}<1 \mathrm{BSs} / \mathrm{m}^{2}$. In particular, the lower and upper bounds are achievable for sufficient expansion and shrinking on $\mathrm{R}_{\mathrm{los}}$ and $\mathrm{R}_{\text {nlos }}$ respectively.

Fig. 6 is consistent with Proposition 3 and 4. In fact, for the purpose to assess the accuracy of $\mathcal{P}_{\mathcal{L}}$ bounds approximation in the optimal regime, we limit first the scaling of $\mathcal{P}_{\mathcal{L}}$ with $\mathrm{T}$ into this regime by considering the combinations $\left(\lambda=\lambda_{g} ; \mathrm{R}_{\text {los }}=\right.$ $\left.1 ; \mathrm{R}_{\text {nlos }}=2\right),\left(\lambda=\frac{\lambda_{f}+\lambda_{g}}{2} ; \mathrm{R}_{\text {los }}=10 ; \mathrm{R}_{\text {nlos }}=20\right)$ and $(\lambda=$ $\left.\lambda_{f} ; \mathrm{R}_{\text {los }}=100 ; \mathrm{R}_{\text {nlos }}=200\right)$, where $\lambda_{f}=\frac{\mathrm{T}^{\delta_{20}}}{\pi F_{-\delta_{20}}(\mathrm{~T})}$ and $\lambda_{g}=\frac{\mathrm{T}_{21}}{\pi F_{-\delta_{21}}(\mathrm{~T})}$. As can be observed from Fig. 6 for $\alpha_{\mathrm{los}}=3$ and $\alpha_{\text {nlos }} \stackrel{\delta_{21}}{=} 4, \lambda_{f}$ and $\lambda_{g}$ are increasing with the SINR target $\mathrm{T}$ until a stage where they become stable and independent from $T$ (Remark 6). Moreover, $\mathcal{P}_{\mathcal{L}}^{\text {SINR }}$ remains bounded by the hypergeometric closed-form expression of $\mathcal{P}_{\mathcal{L}_{1}}^{\text {SINR }}\left(\alpha_{\text {los }} ; \mathrm{T}\right)$ for $\lambda=\lambda_{f}$ and $\mathcal{P}_{\mathcal{L}_{1}}^{\text {SINR }}\left(\alpha_{\text {nlos }} ; \mathrm{T}\right)$ for $\lambda=\lambda_{g}$.

\section{CONCLUSION}

In this paper, we investigated the importance of introducing generalized shadowing and conventional RNPO parameters into the cell-selection model. Using tools from SG, we established an SINR distribution equivalence between a 3D network with shadowing and RNPO parameters and a 2D network in which they are ignored.

Next, for mathematical convenience and model tractability, we proposed an equivalent 2D network based on the $H_{-}$ LOS probability model such as the effect of shadowing and RNPO parameters is interpreted as captured via the fluctuation of aggregated parameters $R_{\text {los }}$ and $R_{\text {nlos. }}$. We derived then the coverage probability and confirmed that its formulation generalizes that of several previous works. Moreover, the regimes where coverage probability is maximized as well as the interference-limited one are investigated based on the scaling of $R_{\text {los }}$ and $R_{\text {nlos }}$, which implicitly reflects different realization of shadowing and RNPO parameters. An intermediary result is a generalisation of the special case closedform expression in [1]. Our results give practical insights for operators and vendors considering the deployment of ultradense $5 \mathrm{G}$ networks.

\section{REFERENCES}

[1] J. G. Andrews, F. Baccelli, and R. K. Ganti, "A tractable approach to coverage and rate in cellular networks," IEEE Trans. Commun., vol. 59, no. 11, pp. 3122-3134, Nov. 2011.

[2] N. Bhushan et al., "Network densification: The dominant theme for wireless evolution into 5G," IEEE Commun. Mag., vol. 52, no. 2, pp. 82-89, Feb. 2014.

[3] I. Atzeni, J. Arnau, and M. Kountouris, "Downlink cellular network analysis with LOS/NLOS propagation and elevated base stations," IEEE Trans. Wireless Commun., vol. 17, no. 1, pp. 142-156, Jan. 2018.

[4] A. Merwaday, R. Vannithamby, M. M. Rashid, Y. Zhang, C. Chen, and $\mathrm{X}$. Wu, "On the performance of directional communications in ultradense networks," in Proc. IEEE Int. Conf. Commun. Workshops (ICC), Paris, France, 2017, pp. 522-527.

[5] R. Hernandez-Aquino, S. A. R. Zaidi, D. McLernon, M. Ghogho, and A. Imran, "Tilt angle optimization in two-tier cellular networks - A stochastic geometry approach," IEEE Trans. Commun., vol. 63, no. 12, pp. 5162-5177, Dec. 2015.

[6] B. Blaszczyszyn, and M. K. Karray, "Spatial distribution of the SINR in Poisson cellular networks with sector antennas," IEEE Trans. Wireless Commun., vol. 15, no. 1, pp. 581-593, Jan. 2016.

[7] H. S. Jo, Y. J. Sang, P. Xia, and J. G. Andrews, "Heterogeneous cellular networks with flexible cell association: A comprehensive downlink SINR analysis," IEEE Trans. Wireless Commun., vol. 11, no. 10, pp. 34843495, Oct. 2012.

[8] J. G. Andrews, X. Zhang, G. D. Durgin, and A. K. Gupta, "Are we approaching the fundamental limits of wireless network densification?" IEEE Commun. Mag., vol. 54, no. 10, pp. 184-190, Oct. 2016.

[9] X. Zhang, and J. G. Andrews, "Downlink cellular network analysis with multi-slope path loss models," IEEE Trans. Commun., vol. 63, no. 5, pp. 1881-1894, May 2015.

[10] F. Baccelli, and B. Blaszczyszyn, "Stochastic Geometry and Wireless Networks: Volume I theory," Found. Trends Netw., vol. 4, nos. 3-4, pp. 249-449, 2010.

[11] B. Blaszczyszyn, and H. P. Keeler, "Equivalence and comparison of heterogeneous cellular networks," in Proc. IEEE 24th Annu. Symp. Pers. Indoor Mobile Radio Commun. Workshops (PIMRC), London, U.K., 2013, pp. 153-157.

[12] P. Madhusudhanan, J. Restrepo, Y. Liu, T. Brown, and K. Baker, "Downlink performance analysis for a generalized shotgun cellular system, IEEE Trans. Wireless Commun., vol. 13, no. 12, pp. 6684-6696, Dec. 2014.

[13] S. Sun, T. A. Thomas, T. S. Rappaport, H. Nguyen, I. Z. Kovacs, and I. Rodriguez, "Path loss, shadow fading, and line-of-sight probability models for 5G urban macro-cellular scenarios," in Proc. IEEE Glob. Conf. Workshops (GLOBECOM), San Diego, CA, USA, 2015, pp. 1-7.

[14] M. Ding, P. Wang, D. Lopez-Perez, G. Mao, and Z. Lin, "Performance impact of LoS and NLoS transmissions in dense cellular networks," IEEE Trans. Wireless Commun., vol. 15, no. 3, pp. 2365-2380, Mar. 2016.

[15] A. P. Prundnikov, Y. A. Brychkov, and O. I. Marichev, Integrals and Series, Vol. 3: More Special Functions, Philadelphia, USA: Gordon and Breach Science Publishers, 1990.

[16] I. S. Gradshteyn,I. M. Ryzhik, Table of Integrals, Series, and Products, 7th ed. Academic Press, Elsevier Inc, 2007.

[17] S. Guruacharya, H. Tabassum and E. Hossain, "Integral approximations for coverage probability," IEEE Wireless Commun. Letters, vol. 5, no. 1, pp. 24-27, Feb. 2016. 